\title{
MITOCHONDRIAL GSH DETERMINES THE TOXIC OR THERAPEUTIC POTENTIAL OF SUPEROXIDE SCAVENGING IN STEATOHEPATITIS
}

\author{
Claudia von Montfort ${ }^{1,2,{ }^{*}}$, Núria Matias ${ }^{1,2,{ }^{*}}$, Anna Fernandez ${ }^{1,2}$, Raquel Fucho ${ }^{1,2}$, Laura \\ Conde de la Rosa ${ }^{1,2}$, Maria Luz Martinez-Chantar ${ }^{2,3}$, José M. Mato ${ }^{2,3}$, Keigo Machida ${ }^{4}$, \\ Hidekazu Tsukamoto ${ }^{4,5}$, Michael P. Murphy ${ }^{6}$, Abdellah Mansouri ${ }^{7}$, Neil Kaplowitz ${ }^{4}$, Carmen \\ Garcia-Ruiz $^{1,2}$, and Jose C. Fernandez-Checa ${ }^{1,2,4}$ \\ ${ }^{1}$ IDIBAPS, Liver Unit-Hospital Clinic, and Department of Cell Death and Proliferation, IIBB-CSIC, \\ 08036-Barcelona, Spain \\ ${ }^{2}$ CIBEREHD, Technology Park of Bizkaia, Bizkaia, Spain \\ ${ }^{3} \mathrm{CIC}$ bioGUNE, CIBEREHD, Technology Park of Bizkaia, Bizkaia, Spain \\ ${ }^{4}$ Southern California Research Center for ALPD and Cirrhosis, Keck School of Medicine of the \\ University of Southern California \\ ${ }^{5}$ Department of Veterans Affairs Greater Los Angeles Healthcare System, Los Angeles, CA, USA \\ ${ }^{6}$ MRC Mitochondrial Biology Unit, Hills Road, Cambridge CB2 OXY, UK \\ ${ }^{7}$ Centre de Recherche Bioméicale Bichat Beaujon, Université Paris 7 Denis Diderot, F-75018, \\ Paris, France
}

\section{Abstract}

Background \& Aim-Steatohepatitis ( $\mathrm{SH}$ ) is associated with mitochondrial dysfunction and superoxide overgeneration, which can be converted into $\mathrm{H}_{2} \mathrm{O}_{2}$ by SOD2. Since mitochondrial GSH (mGSH) plays a critical role in $\mathrm{H}_{2} \mathrm{O}_{2}$ reduction, we explored the interplay between superoxide, $\mathrm{H}_{2} \mathrm{O}_{2}$ and mGSH in nutritional and genetic models of $\mathrm{SH}$, which exhibit $\mathrm{mGSH}$ depletion.

\begin{abstract}
Methods-Isolated mitochondria and primary hepatocytes, as well as in vivo SH models showing mGSH depletion to test the consequences of superoxide scavenging.

Results-In isolated mitochondria and primary hepatocytes superoxide scavenging by SOD mimetics or purified SOD decreased superoxide and peroxynitrite generation but increased $\mathrm{H}_{2} \mathrm{O}_{2}$ following mGSH depletion despite mitochondrial peroxiredoxin/thioredoxin defense. Selective mGSH depletion sensitized hepatocytes to cell death induced by SOD mimetics and this was prevented by RIP1 kinase inhibition with necrostatin-1 or GSH repletion with GSH ethyl ester (GSHee). Mice fed the methinonie-choline deficient (MCD) diet or MAT1 $\mathrm{A}^{-/-}$mice exhibit reduced SOD2 activity; in vivo treatment with SOD mimetics increased liver damage,
\end{abstract}

(C) 2012 European Association of the Study of the Liver. Published by Elsevier B.V. All rights reserved.

Correspondence: JoséC. Fernádez-Checa, Center Esther Koplowitz, C/Rosello 149-153, 08036-Barcelona; checa229@ yahoo.com. *laudia von Montfort and Nuria Matís contributed equally to the work.

Publisher's Disclaimer: This is a PDF file of an unedited manuscript that has been accepted for publication. As a service to our customers we are providing this early version of the manuscript. The manuscript will undergo copyediting, typesetting, and review of the resulting proof before it is published in its final citable form. Please note that during the production process errors may be discovered which could affect the content, and all legal disclaimers that apply to the journal pertain. 
inflammation, and fibrosis, despite decreased superoxide and 3-nitrotyrosine immunoreactivity, effects that were ameliorated by mGSH replenishment with GSHee but not NAC. As a proof-ofprinciple of the detrimental role of superoxide scavenging in the face of mGSH depletion, transgenic mice overexpressing SOD2 exhibited enhanced susceptibility to MCD-mediated SH.

Conclusion-These findings underscore a critical role for mGSH in the therapeutic potential of superoxide scavenging in $\mathrm{SH}$, and suggest that the combined approach of superoxide scavenging with mGSH replenishment may be of significance in SH.

\section{INTRODUCTION}

Steatohepatitis (SH) is an intermediate stage of alcoholic (ASH) and nonalcoholic steatohepatitis (NASH), which can further progress to cirrhosis and hepatocellular carcinoma. Predominant etiology includes alcohol drinking or insulin resistance/type II diabetes. Despite intense research ASH/NASH pathogenesis is still poorly understood [1]. Mitochondrial dysfunction and oxidative stress are critical players, taking a central stage in the "two-hits" scenario of SH [2]. Although other potential mechanisms contribute to disease progression (e.g. endoplasmic reticulum stress), NASH is considered a mitochondrial disease [3]. Due to the rising prevalence of obesity worldwide NASH constitutes a global health concern requiring the urgent need for more effective therapies.

Mitochondria are the primary intracellular sites of oxygen consumption and the major source of reactive oxygen species (ROS) generation is the mitochondrial respiratory chain (MRC) [4]. Patients with NASH exhibit defective MRC [5], and increased mitochondrial generation of superoxide has been shown in ASH/NASH models [6, 7]. In addition to its own damaging reactivity with iron-sulfur centers, the superoxide can be the source of further ROS and reactive nitrogen species (RNS). Superoxide can interact with nitric oxide (NO) to produce the highly reactive product peroxynitrite, which has a short half-life and is responsible for many damaging modifications of tyrosine residues in mitochondrial target proteins [8]. The elimination of mitochondrial superoxide by SOD2 is essential in the defense against oxidative stress and SOD2 deficiency leads to neonatal death in mice. The dismutation of superoxide generates $\mathrm{H}_{2} \mathrm{O}_{2}$, a potent oxidant. The degradation of mitochondrial $\mathrm{H}_{2} \mathrm{O}_{2}$ is carried out by the antioxidant enzymes GSH peroxidase 1 (GSHPx1) and peroxiredoxin-III (Prx-III), the former of which requires GSH for its activity. Prx-III reactivation upon reduction of $\mathrm{H}_{2} \mathrm{O}_{2}$ occurs by thioredoxin-2 (Trx2). The status of SOD2 in $\mathrm{SH}$ is not well established with studies indicating either decreased or no changes in expression $[9,10]$. Moreover, mitochondrial GSH (mGSH) depletion has been reported in both ASH/NASH models [7] as well as in human NASH [11].

Given the role of superoxide in pathogenesis, its scavenging may be of significance in diverse diseases. To overcome the problems associated with the use of the native SOD enzymes, such as their inaccessibility to intracellular compartments and adverse effects due to immunogenic responses, chemical SOD mimetics have been developed and characterized for SOD activity [12] and shown in vivo therapeutic potential against a wide range of diseases [13]. However, studies using SOD mimetics in SH are scarce, and the interplay between superoxide scavenging, $\mathrm{H}_{2} \mathrm{O}_{2}$ and $\mathrm{mGSH}$ in disease progression has not been specifically examined. Thus, we addressed the role of $\mathrm{mGSH}$ in controlling the therapeutic potential of superoxide scavenging in nutritional and genetic NASH models namely, MAT1A knockout mice [14] and mice fed methionine-choline deficient (MCD) diet [15], which exhibit mGSH depletion [16]. Our findings indicate that SH is characterized by the impairment of both SOD2 and mGSH defense. However, the beneficial effect of superoxide scavenging is determined by $\mathrm{mGSH}$, and hence their combination may be of relevance in $\mathrm{SH}$. 


\section{MATERIALS AND METHODS}

\section{Animals, mitochondria isolation and treatments}

Male mice (C57BL/6J, 8 wks) (Charles River Laboratories) were housed in a pathogen-free barrier facility and the protocols approved by the IDIBAPS Animal Care Committee. Mice were fed MCD diet (or the corresponding control diet supplemented with methionine and choline), alcohol liquid diet (intragastrically or orally for 1-12 months) as described [17, 18], or a high fat diet (60\% of the KCal derived from fat, Research Diets) for 16-20 weeks. TgSOD2 mice have been characterized before [19] and expressed 2-3 fold SOD2 levels compared to wild type mice. Rat liver mitochondria were isolated as described previously [20], and mitochondrial purity was assessed by determination of succinic dehydrogenase, lactic dehydrogenase and the presence of microsomes (GRP78/Bip), plasma membrane (Na +/K+ ATPase), endosomes (Rab5A/Rab11) and lysosomes (Lamp1). In addition, we monitored the acceptor control ratio (ACR) as a measure of mitochondrial quality. Furthermore, isolated mitochondria and hepatocytes were prepared from mice fed MCD or MAT1A null mice to determine the generation of superoxide and hydrogen peroxide and cell death. In other experiments superoxide anion was generated in isolated mitochondria by xanthine/xanthine oxidase $(\mathrm{X} / \mathrm{XO}, 0.1 \mathrm{mM} / 20 \mathrm{U} / \mathrm{ml})$, glucose/glucose oxidase $(\mathrm{G} / \mathrm{GO}$, $0.1 \mathrm{mM} / 20 \mathrm{U} / \mathrm{ml})$ in the presence or absence of MnTBAP $(50 \mathrm{M})$, CuDIPS (50 M) or purified SOD from bovine erythrocytes (Sigma) with or without mGSH depletion. mGSH depletion in isolated mitochondria was induced by ethacrinic acid (EA, $250 \mathrm{M})$ or diethylmaleate (DEM, $0.8 \mathrm{mM}$ ) and with 3-hydroxy-4-pentenoate (HP, 1mM) in hepatocytes as described (20). In some cases, hepatocytes were incubated with necrostatin-1 (20 M), caspase inhibitor I (Calbiochem, $50 \mathrm{M})$, desferoxamine $(0.5 \mathrm{mg} / \mathrm{ml})$ or catalase $(200 \mathrm{U} / \mathrm{ml})$ to determine cell viability.

\section{ROS/RNS determination}

ROS determination was measured by MitoSOX ( $5 \mu \mathrm{M}$, Molecular Probes) or Amplex Red Hydrogen peroxide/Peroxidase Assay Kit (1 M, Invitrogen) as described before [21] and detailed in Supplemental Methods. In some cases, mitochondria were incubated with the nitric oxide donor Deta NoNoate and peroxynitrite was determined using the specific probe APF (5 M, Molecular Probes) which does not monitor NO, superoxide or hydrogen peroxide [22].

\section{In vivo effects of SOD mimics in nutritional and genetic NASH models}

Mice were fed a MCD diet (2-15 days) with or without MnTBAP treatment (10 g/kg, ip) for 2 days. In addition, MAT1A null mice ( 3 and 9 months old) or corresponding wild type mice were treated with MnTBAP for 2 days. Serum and liver samples were processed for ALT and H\&E staining. Inflammatory cell infiltration was examined by myeloperoxidase staining, and Sirius red staining was used to monitor fibrosis. In some cases, MCD-fed mice were co-treated with GSHee, NAC or necrostatin-1 to evaluate the course of liver damage induced by superoxide scavenging with MnTBAP. Lipid peroxidation was assessed as the presence of carbonyl proteins determined by immunoblotting as described before [23]. In addition, in some cases MCD-fed mice were treated with the mitochondrial targeted antioxidant MitoQ or its inactive analogue decylT

\section{Statistical analyses}

Results are reported as means \pm SD. Student's t-test or ANOVA with Bonferroni's post-hoc test or Mann-Whitney Rank Sum test was used for the determination of statistical significance among treatment groups, as appropriate. 


\section{RESULTS}

\section{Mitochondrial superoxide scavenging increases $\mathrm{H}_{2} \mathrm{O}_{2}$ following GSH depletion despite Prx-III/Trx2 defense}

To test the hypothesis that GSH is critical for the fate of $\mathrm{H}_{2} \mathrm{O}_{2}$ derived from superoxide scavenging, we examined their interplay in isolated mitochondria with or without GSH depletion using well-characterized electrophiles. EA depleted mitochondrial GSH levels by 70-80\% (Figure 1A). To mimic the superoxide generation occurring in $\mathrm{SH}, \mathrm{X} / \mathrm{XO}$ was used to increase superoxide anion and $\mathrm{H}_{2} \mathrm{O}_{2}$ (Figure $1 \mathrm{~B}, \mathrm{C}$ ). The addition of the SOD mimetic MnTBAP significantly decreased superoxide formation by $\mathrm{X} / \mathrm{XO}$, leading to a slight increase in $\mathrm{H}_{2} \mathrm{O}_{2}$ levels (Figure 1B, C). MnTBAP alone, however, did not increase the basal levels of superoxide, eliminating a direct role for the SOD mimetic in ethidium oxidation. To validate the effects of MnTBAP on superoxide scavenging, we used purified SOD from bovine erythocytes, which recapitulated the effects on superoxide and $\mathrm{H}_{2} \mathrm{O}_{2}$ induced by MnTBAP (Supplementary Figure 1A, 1B). However, in mitochondria that have been GSHdepleted by EA treatment, MnTBAP caused opposite quantitative changes, decreasing superoxide but increasing hydrogen peroxide following $\mathrm{X} / \mathrm{XO}$ exposure (Figure $1 \mathrm{C}$ ). The addition of exogenous catalase abolished the increase of $\mathrm{H}_{2} \mathrm{O}_{2}$ stimulated by MnTBAP in GSH depleted mitochondria (Figure 1C). Similar findings were observed when GSH was depleted in isolated mitochondria by DEM (Supplementary Figure 2A, B) or after in vivo treatment with buthionine sulfoximine (not shown). Moreover, similar to mitochondria exposed to $\mathrm{X} / \mathrm{XO}$ increased superoxide anion and hydrogen peroxide levels were observed in isolated mitochondria from mice fed MCD compared to mitochondria from control mice fed methionine and choline supplemented diet (Supplementary Figure 3).

In addition to GSH, Prx-III also plays a key role in controlling the levels of mitochondrial $\mathrm{H}_{2} \mathrm{O}_{2}$ [7]. We monitored the redox status of Prx-III in isolated mitochondria with or without AMS, which modifies reduced protein thiols [24]. Compared to tert-butylhydroperoxide treatment, Prx-III remained in the reduced state despite GSH depletion by EA as seen by the slower migration induced by AMS compared to oxidized form (Figure 1D). Trx 2 activity (Supplementary Figure 4A) remained unchanged regardless of GSH depletion or not. However, most of Prx-III was in the oxidized form following inhibition of Trx-2 with auronofin [25], as AMS did not affect Prx-III mobility (Supplementary Figure 4B). Thus, GSH plays a critical role in preventing the collateral accumulation of $\mathrm{H}_{2} \mathrm{O}_{2}$ following superoxide scavenging by SOD mimetics.

\section{Mitochondrial peroxynitrite generation requires exogenous NO source and decreases upon superoxide scavenging}

Superoxide can react with NO to yield peroxynitrite. Since uric acid scavenges peroxynitrite and is produced from xanthine oxidation, we used G/GO to generate superoxide and examined peroxynitrite with or without GSH depletion. The generation of peroxynitrite in liver mitochondria, measured by the specific APF probe [22] was negligible unless supplemented with the NO donor Deta NoNoate (Supplementary Figure 5). Purified SOD decreased peroxynitrite levels induced by DetaNoNoate and G/GO to a similar extent regardless of GSH status (Supplementary Figure 5). Thus, mitochondrial peroxynitrite formation requires an exogenous source of $\mathrm{NO}$ and is abolished by superoxide scavenging.

\section{Selective mGSH depletion sensitizes hepatocytes to SOD mimetics-induced cell death}

Next, we assessed the effect of SOD mimetics in isolated hepatocytes with mGSH depletion by HP as described [20, 24]. HP depleted mGSH levels but spared cytosolic GSH (Figure 1E). To stimulate mitochondrial superoxide, hepatocytes were incubated with antimycin $A$ (AA, $15 \mathrm{M}$ ) to block electron transfer at complex III of the MRC [26]. AA increased 
superoxide generation (Figure 1F). MnTBAP reduced AA-induced superoxide levels and slightly increased those of $\mathrm{H}_{2} \mathrm{O}_{2}$ (Figure $1 \mathrm{~F}, \mathrm{G}$ ), with similar findings observed with CuDIPS (not shown). In mGSH-depleted hepatocytes by HP, MnTBAP reduced the generation of superoxide induced by AA, but enhanced the formation of $\mathrm{H}_{2} \mathrm{O}_{2}$ (Figure 1F, $\mathrm{G})$. This outcome was associated with sustained activation of the stress kinase JNK, compared to its transient activation in mGSH-repleted cells (Figure 1H) and with increased cell death (Figure 1I), characterized by Sytox green staining (Supplementary Figure 6) and lack of nuclear fragmentation (not shown). Furthermore, HP-mediated sensitization of hepatocytes to superoxide scavenging was not due to altered mitochondrial protein thiols using the thiol reactive compound (4-iodobutyl)triphenylphosphonium (not shown), in agreement with previous observations [24]. Moreover, hepatocytes from MCD fed mice, which exhibit mGSH depletion and reduced SOD2 activity (see below), exhibited intrinsinc cell death and were more sensitive to AA than corresponding control hepatocytes (Figure 1J). Similar findings were observed in hepatocytes from MAT1A null mice (not shown). These findings establish a critical role for mGSH in the safety of superoxide scavenging by SOD mimetics in hepatocytes.

\section{Protection of hepatocytes against superoxide scavenging following $\mathrm{mGSH}$ depletion}

Consistent with the lack of caspase activation (Supplementary Figure 7), a pancaspase inhibitor (caspase inhibitor I, Calbiochem), which prevents Fas and staurosporine induced cell death, failed to protect mGSH-depleted hepatocytes from AA+MnTBAP exposure following mGSH depletion (Figure 1I). Moreover, cyclosporine A, which inhibits mitochondrial membrane permeability transition (MPT), protected against AA+MnTBAPmediated killing of hepatocytes following mGSH depletion (Figure 1I). In addition, GSHee, a permeable GSH form that restores mGSH [24], rescued HP-treated hepatocytes from AA + MnTBAP-mediated cell death (Figure 1I). Importantly, catalase protected hepatocytes that have been mGSH depleted either by HP or MCD feeding from AA stimulated cell death (Figure 1I, J), indicating the involvement of overgeneration of hydrogen peroxide in this event. Pretreatment with the iron chelator desferoxamine protected mGSH-depleted hepatocytes against cell death induced by AA+MnTBAP (Figure 1I). Moreover, since RIP1 kinase plays a pivotal role in necrotic cell death, we tested the effect of necrostatin-1, a RIP1 kinase inhibitor [27]. As seen, necrostatin-1 protected mGSH-depleted hepatocytes against AA+MnTBAP-induced cell death (Figure 1I). Interestingly, necrostatin-1 did not abolish the sustained JNK activation by MnTBAP in GSH-depleted hepatocytes (Figure 1H), consistent with previous findings [28]. Moreover, SP600125 a JNK inhibitor failed to protect hepatocytes against superoxide scavenging following HP sensitization (not shown).

\section{Decreased SOD2 activity in experimental SH}

Since the status of SOD2 in NASH is not well established, we examined its expression and activity in nutritional and genetic NASH models $[14,15]$. We chose the MCD feeding model since although it does not induce insulin resistance it does reproduce key features of human NASH such as oxidative stress, hepatocellular death, inflammation and fibrosis [15], which reflects disease progression [29]. For genetic models, we used MAT1A null mice, which exhibit SH and spontaneous hepatocarcinoma at advanced age [14, 30]. We avoided the obese ob/ob mice because of the unestablished and controversial status of SOD2 due in part to altered $\mathrm{Mn}^{2+}$ metabolism [9,31-33]. While mRNA SOD2 levels increased in both MCD-fed mice and MAT1 $\mathrm{A}^{-/}$mice (Figure 2), this outcome did not translate in enhanced SOD2 activity (Figure 2). Samples from mice fed alcohol for 12 months but not 4 weeks exhibited increased SOD2 expression (Supplementary Figure 8) but decreased activity. Taken together SOD2 activity is decreased in nutritional and genetic models of SH. 


\section{SOD mimetics exacerbate liver damage in nutritional and genetic SH models}

To test the in vivo relevance, we examined the effect of mGSH depletion on superoxide scavenging in nutritional and genetic SH models $[14,15,30]$. MCD feeding depleted mGSH levels (Supplementary Figure 9A) by decreasing mitochondrial membrane fluidity [16]. In MAT1 $^{-/-}$mice mGSH depletion (Figure 3A) reflects the cytosol GSH limitation due to decreased SAM levels [14]. Treatment of 3-months old MAT1A mice with MnTBAP (10 g/ mice, i.p) decreased hepatic superoxide but enhanced hydrogen peroxide (Figure 3B, C), with similar findings in MCD-fed mice (Supplementary Figure 9B). Interestingly, although SH symptoms occur in MAT1 $\mathrm{A}^{-/-}$mice after 3 months of age [14], MnTBAP treatment enhanced liver damage in the MAT1 $\mathrm{A}^{-/-}$mice as revealed by increased ALT release (Figure 3D) and histological analysis (Supplementary Figure 10), with similar findings in MCD-fed mice (Supplementary Figure 9C, 9D). MnTBAP worsened inflammation (Figure 3E) and Sirius red staining (Figure 3F) and hydroxyproline (not shown) in 3-months old MAT1 ${ }^{-/-}$ mice and MCD-fed mice (Supplementary Figure 9E, 9F), without an effect on steatosis (Supplementary Figure 11A, B). Paralelled to the increased MPO and Sirius red staining in MCD-fed mice treated with MnTBAP, we observed increased expression of MCP1, IL-1 and TGF- by quantitative PCR analysis (Supplementary Figure 12). Similar findings were observed in mice fed a HFD for 16-20 weeks following MnTBAP treatment (not shown). Interestingly, these adverse events induced by MnTBAP occurred despite lower 3nitrotyrosine immunohistochemistry in both $\mathrm{MAT}^{-1 \mathrm{~A}^{-/}}$mice (Figure 3G) and MCD-fed mice (Supplementary Figure 9G). To ensure the oxidant-dependent nature of the preceding events, we tested the role of mitochondrial-targeted antioxidant MitoQ [34]. In contrast to decylTPP, in vivo treatment with MitoQ reduced MnTBAP potentiation of MCD-induced liver injury, fibrosis and inflammation (Supplementary Figure 13). Thus, these findings underscore that in vivo treatment with the SOD mimetic MnTBAP worsens SH despite reduced superoxide levels and nitrosative stress.

\section{mGSH replenishment and necrostatin-1 protect MCD-fed mice from liver damage potentiated by MnTBAP}

To address whether the enhanced susceptibility to MnTBAP was due to mGSH depletion, we compared the therapy of GSHee vs. NAC in MCD-fed mice. GSHee restored the mGSH levels in MCD-fed mice (Figure 3H). In contrast, while NAC, a GSH precursor, maintained cytosol GSH it failed to boost the mGSH levels (Figure 3H, I), reflecting the impairment of mGSH transport induced by MCD [16]. Furthermore, GSHee protected MCD-fed mice from MnTBAP-induced liver damage revealed by ALT (Figure 3J) and histological analysis (not shown). Consistent with the findings in GSH compartimentalization, NAC was inefficient in protecting MCD-fed mice from MnTBAP-mediated liver damage (Figure 3J). Moreover, necrostatin-1, which protected mGSH-depleted hepatocytes against AA+MnTBAP-induced cell death, rescued MCD-fed mice against MnTBAP-mediated liver injury (Figure 3K). Thus, these findings indicate that the worsening by SOD mimetics of nutritional SH is due largely to mGSH depletion.

\section{Transgenic SOD2 mice exhibit increased susceptibility to MCD-induced steatohepatitis}

To validate the preceding findings and to discard unspecific effects of chemical SOD mimetics, we used TgSOD2 mice to test their susceptibility to MCD. Although the basal levels of $\mathrm{mGSH}$ in wild type and TgSOD2 did not differ $(5.8 \pm 0.7$ and $6.1 \pm 0.8 \mathrm{nmol} / \mathrm{mg}$ prot, respectively), MCD feeding depleted mGSH in both mice to a similar extent ( $42 \%$ and $48 \%$ respectively). However, the TgSOD2 mice exhibited more liver injury than wild type mice upon MCD feeding as seen by serum ALT release (Figure 4A) and H\&E imaging (not shown). Moreover, accompanying these observations, the degree of fibrosis (Figure 4B) and inflammation (Figure 4C) increased in TgSOD2 mice compared to wild type mice following MCD feeding. In contrast, 3-nitrotyrosine immunoreactivity, reflecting peroxynitrite 
generation, was lower in TgSOD2 mice with respect to wild type mice after MCD feeding

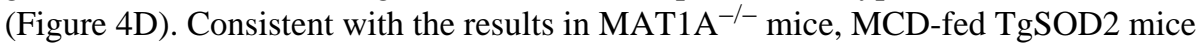
exhibited decreased superoxide levels but enhanced hydrogen peroxide levels and were protected by GSHee treatment (not shown). Thus, these findings show that overexpression of SOD2 in the face of mGSH depletion sensitizes to nutritional SH.

\section{Discussion}

Mitochondrial dysfunction is a prominent feature of SH and contributes to the superoxide generation and other ROS/RNS species. Here we show that nutritional and genetic models of SH exhibit decreased SOD2 activity and mGSH depletion. Since the product of SOD2, $\mathrm{H}_{2} \mathrm{O}_{2}$, is the substrate of the GSH redox cycle, we examined the interplay between superoxide scavenging and mGSH-dependent $\mathrm{H}_{2} \mathrm{O}_{2}$ generation in mice fed MCD as well as MAT1 ${ }^{-/-}$mice. In addition to SOD2, $\mathrm{H}_{2} \mathrm{O}_{2}$ can be also generated from other mechanisms within mitochondria, including the action of $\mathrm{p} 66^{\mathrm{Shc}}$, which directly stimulates hydrogen peroxide generation by transferring electrons to cytochrome $\mathrm{c}$ [35]. Thus, mitochondrial hydrogen peroxide homeostasis reflects the balance between its generation and its reduction, mainly via the GSH redox cycle. While mGSH depletion in either model occurs via different mechanisms, including limited cytosol GSH availability and altered mitochondrial membrane dynamics [16], enhanced SOD2 expression does not correlate with activity. Decreased SOD2 activity is observed in either model and may reflect sensitivity to oxidative/nitrosative stress. For instance, SOD2 contains tyrosine residues that are susceptible to be nitrated or oxidized by peroxynitrite, leading to enzymatic inactivation [36, 37]. Consistent with impaired SOD2 activity, both models show enhanced basal levels of superoxide. Since superoxide can react with NO to form peroxynitrite, a reactive nitrogen species responsible for mitochondrial dysfunction [8], the use of SOD mimetics would imply a dual benefit, diminishing both superoxide and peroxynitrite. Contrary to these expectations, superoxide scavenging sensitized hepatocytes to oxidant-dependent necrosis following mGSH depletion due to increased $\mathrm{H}_{2} \mathrm{O}_{2}$. The toxic effect of enhanced $\mathrm{H}_{2} \mathrm{O}_{2}$ could be due to the generation of hydroxyl radical via Fenton reaction (Figure 4E) and the induction of MPT by targeting critical cysteine resides in cyclophilin D [38]. Consistent with these possibilities, the iron chelator desferoxamine or cyclosporine A protected mGSHdepleted hepatocytes against superoxide anion scavenging. Moreover, treatment with MnTBAP of both MCD-fed mice and 3-months old MAT1A ${ }^{-/-}$mice, which does not yet exhibit signs of SH [14], increased and accelerated signs of disease progression (hepatocellular damage, inflammation and fibrosis) despite reducing superoxide and 3nitrotyrosine immunoreactivity. While these effects were mitigated by recovery of the mGSH store by GSHee, NAC failed to recover the mGSH pool and to protect both models against MnTBAP-enhanced pathology. Our findings are consistent with previous reports defining a critical role for mGSH in the control of stimulated $\mathrm{H}_{2} \mathrm{O}_{2}$ generation in the MRC [26, 39]. Low levels of mGSH depletion (up to $40 \%$ ) had little effect on $\mathrm{H}_{2} \mathrm{O}_{2}$ diffusion from mitochondria. However, GSH depletion below a critical threshold (about 50\%) results in a linear increase in $\mathrm{H}_{2} \mathrm{O}_{2}$ production from mitochondria, which compromises cell viability and mitochondrial function [26, 39].

Chemical SOD mimetics have been developed and characterized for SOD activity [12, 13]. In particular the anionic porphyrin MnTBAP has been shown to exhibit scavenging activity towards superoxide anion and peroxynitrite [40]. While MnTBAP was inefficient in supporting the aerobic growth of SOD-deficient E. coli [40], however studies in Sod2 ${ }^{\mathrm{tm} 1 \mathrm{Cje}}$ null mice demonstrated the therapeutic benefit of MnTBAP administration, including prevention of systemic pathology such as dilated cardiomyopathy, hepatic lipid accumulation, and most importantly, a dramatically extended lifespan [41]. Thus, these data with the SOD2 null mice indicate that this particular SOD mimetic can functionally 
overcome SOD2 deficiency. Regardless of whether MnTBAP is specific for scavenging superoxide anion or peroxynitrite, since the latter derives from the former upon reaction with NO, the elimination of superoxide anion will invariably result in decreased peroxynitrite as well. Moreover, our findings with MnTBAP in isolated mitochondria were validated with purified SOD. More importantly, the increased susceptiblity of TgSOD2 mice to MCD-induced liver injury, inflammation and fibrosis provide compelling evidence for the deleterious effects of superoxide scavenging in the face of mGSH depletion.

Our findings have potential clinical implications as they may help explain the failure of antioxidant treatment so far in human liver diseases. Data and models described here could offer an explanation for the important discrepancy between the overwhelming evidence for an important role of ROS and the common clinical failure of trials. Thus, this study indicates that a better approach to treat SH is the combination of SOD mimics with mGSH restoration rather than improving only SOD2 with SOD mimetics, illustrating the importance of a delicate equilibrium between these two antioxidants to ensure protection and therapeutic benefit.

\section{Supplementary Material}

Refer to Web version on PubMed Central for supplementary material.

\section{Acknowledgments}

This work was carried out (in part) at the Esther Koplowitz Centre. The technical assistance of Susana Nuñz in the in vivo studies is greatly appreciated. The authors thank Dr. C. SanMartin (University of Navarra) for synthesizing HP.

Financial support: The work was supported by grants SAF2008-02199, SAF2008-04800 and SAF2009-11417, SAF2011-23031 (Plan Nacional de I+D), by CIBEREHD from the Instituto Carlos III, the Fundació Mutua Madrileña, the NIH grants R01AT1576, R01 AA018857, and the center grant P50AA11999 (Southern California Research Center for ALPD and Cirrhosis) from the National Institute on Alcohol Abuse and Alcoholism of NIH, USA, FundacióLa Marató de TV3, and Medical Research Service of Department of Veterans Affairs.

\section{ABBREVIATIONS}

$\begin{array}{ll}\text { ASH } & \text { alcoholic steatohepatitis } \\ \text { AA } & \text { antimycin A } \\ \text { HP } & \text { 3-hydroxy-4-pentenoate } \\ \text { GSHee } & \text { GSH ethyl ester } \\ \text { GSHPx1 } & \text { GSH peroxidase 1 } \\ \text { mGSH } & \text { mitochondrial GSH } \\ \text { MCD } & \text { methionine-choline deficient diet } \\ \text { MRC } & \text { mitochondrial respiratory chain } \\ \text { NAC } & \text { N-acetylcysteine } \\ \text { NASH } & \text { nonalcoholic steatohepatitis } \\ \text { SH } & \text { steatohepatitis } \\ \text { Prx-III } & \text { peroxiredoxin-III } \\ \text { SOD } & \text { superoxide dismutase }\end{array}$



ROS
reactive oxgen species
RNS
reactive nitrogen species
Trx2
thioredoxin-2.

\section{REFERENCES}

1. Tilg H, Diehl MA. Citokines in alcoholic and nonalcoholic steatohepatitis. N. Engl. J. Med. 2000; 343:1467-1476. [PubMed: 11078773]

2. Day CP, James OF. Steatohepatitis: a tale of two "hits"? Gastroenterology. 1998; 114:842-845. [PubMed: 9547102]

3. Pessayre D, Fromenty B. NASH: a mitochondrial disease. J Hepatol. 2005; 42:928-940. [PubMed: 15885365]

4. Balaban RS, Nemoto S, Finkel T. Mitochondria, oxidants, and aging. Cell. 2005; 120:483-495. [PubMed: 15734681]

5. Pérez-Carreras M, et al. Defective hepatic mitochondrial respiratory chain in patients with nonalcoholic steatohepatitis. Hepatology. 2003; 38:999-1007. [PubMed: 14512887]

6. Larosche I, et al. Prolonged ethanol administration depletes mitochondrial DNA in MnSODoverexpressing transgenic mice, but not in their wild type littermates. Toxicol Appl Pharmacol. 2009; 234:326-338. [PubMed: 19063909]

7. Marí M, et al. Redox control of liver function in health and disease. Antioxid Red Signal. 2010; 12:1295-1331.

8. Garcia-Ruiz I, et al. Mitochondrial Complex I Subunits Are Decreased in Murine Nonalcoholic Fatty Liver Disease: Implication of Peroxynitrite research articles. J Proteom Res. 2010; 48:24502459.

9. Laurent A, et al. Pivotal role of superoxide anion and beneficial effect of antioxidant molecules in murine steatohepatitis. Hepatology. 2004; 39:1277-1285. [PubMed: 15122756]

10. Perlemuter G, et al. Increase in liver antioxidant enzyme activities in non-alcoholic fatty liver disease. Liver international. 2005; 25:946-953. [PubMed: 16162151]

11. Serviddio G, et al. Uncoupling protein-2 (UCP2) induces mitochondrial proton leak and increases susceptibility of non-alcoholic steatohepatitis (NASH) liver to ischaemia-reperfusion injury. Gut. 2008; 57:957-965. [PubMed: 18308829]

12. Salvemini D, Riley DP, Cuzzocrea S. SOD mimetics are coming of age. Nat Rev Drug Discovery. 2002; 1:367-374.

13. Batinić-Haberle I, Rebouças JS, Spasojević I. Superoxide dismutase mimics: chemistry, pharmacology, and therapeutic potential. Antioxid Redox Signal. 2010; 13:877-918. [PubMed: 20095865]

14. Lu SC, et al. Methionine adenosyltransferase 1A knockout mice are predisposed to liver injury and exhibit increased expression of genes involved in proliferation. Proc Natl Acad Sci USA. 2001; 98:5560-5565. [PubMed: 11320206]

15. Hebbard L, George J. Animal models of nonalcoholic fatty liver disease. Nat Rev. Gastroenterol \& hepatol. 2011; 8:35-44. [PubMed: 21119613]

16. Caballero F, et al. Specific contribution of methionine and choline in nutritional nonalcoholic steatohepatitis: impact on mitochondrial S-adenosyl-L-methionine and glutathione. J Biol Chem. 2010; 285:18528-18536. [PubMed: 20395294]

17. Deng Q-G, et al. Steatohepatitis induced by intragastric overfeeding in mice. Hepatology. 2005; 42:905-914. [PubMed: 16175602]

18. Ji C, Mehrian-Shai R, Chan C, Hsu Y, Kaplowitz N. Role of CHOP in hepatic apoptosis in the murine model of intragastric ethanol feeding. Alcohol Clin Exp Res. 2008; 29:1496-1503. [PubMed: 16131858] 
19. Raineri L, et al. Strain dependent high level expression of a transgene for mangenese superoxide dismutase is associated with growth retardation and decreased fertility. Free Radical. Biol. Med. 2001; 31:1018-1030. [PubMed: 11595386]

20. Marí M, et al. Mitochondrial free cholesterol loading sensitizes to TNF- and Fas-mediated steatohepatitis. Cell Metab. 2006; 4:185-198. [PubMed: 16950136]

21. Bell E, Klimova T, Eisenbart J, Moraes C, Murphy M, et al. The Qo site of the mitochondrial complex III is required for the transduction of hypoxic signaling via reactive oxygen species production. J. Cell Biol. 2007; 177:1029-1036. [PubMed: 17562787]

22. Kondrikov D, et al. Beta-actin association with endothelial nitric-oxide synthase modulates nitric oxide and superoxide generation from the enzyme. J Biol Chem. 2010; 285:4319-4327. [PubMed: 19946124]

23. Fernández A, Llacuna L, Fernández-checa JC, Colell A. Mitochondrial cholesterol loading exarcebates amyloid beta peptide-induced inflammation and neurotoxicity. J Neurosc. 2009; 29:6394-6640.

24. Marí M, et al. Mechanism of mitochondrial glutathione-dependent hepatocellular susceptibility to TNF despite NF-kappaB activation. Gastroenterology. 2008; 134:1507-1520. [PubMed: 18343380]

25. Lowes D, Galley HF. Mitochondrial protection by the thioredoxin-2 and glutathione systems in an in vitro endothelial model of sepsis. Biochem J. 2011; 436:123-132. [PubMed: 21355852]

26. García-Ruiz C, Colell A, Morales A, Kaplowitz N, Fernández-Checa J. Role of oxidative stress generated from the mitochondrial electron transport chain and mitochondrial glutathione status in loss of mitochondrial function and activation of transcription factor nuclear factor-kappa B: studies with isolated mitochondria and. Mol Pharmacol. 1995; 48:825-834. [PubMed: 7476912]

27. Degterev A, et al. Chemical inhibitor of nonapoptotic cell death with therapeutic potential for ischemic brain injury. Nat Chem Biol. 2005; 1:112-119. [PubMed: 16408008]

28. Degterev A, et al. Identification of RIP1 kinase as a specific cellular target of necrostatins. Nat Chem Biol. 2008; 4:313-321. [PubMed: 18408713]

29. Elizabeth M, Brunt MD. Nonalcoholic steatohepatitis in children: a multicenter clinicopathological study. Hepatology. 2009; 50:1113-1120. [PubMed: 19637190]

30. Mato JM, Martinez-Chantar ML, Lu SC. Methionine Metabolism and Liver Disease. Annual Review of Nutrition. 2008; 28:273-293.

31. Ozata M, Uckaya G, Aydin A, Isimer A, Ozdemir IC. Defective antioxidant defense system in patients with a human leptin gene mutation. Hormone and metabolic research. 2000; 32:269-272. [PubMed: 10965932]

32. Welsh JJ, Narbaitz R, Begin-heick N. Metabolie Effects of Dietary Manganese mentation in ob / ob Mice. J Nutri. 1985; 115:919-928.

33. Yang S, et al. Mitochondrial adaptations to obesity-related oxidant stress. Arch Biochem Biophys. 2000; 378:259-268. [PubMed: 10860543]

34. Murphy MP, Smith RAJ. Ann Rev Pharmacol Toxicol. 2011; 47:629-656.

35. Giorgio, et al. Electron transfer between cytochrome $\mathrm{c}$ and p66Shc generates reactive oxygen species that trigger mitochondrial apoptosis. Cell. 2005; 122:221-233. [PubMed: 16051147]

36. Demicheli V, Quijano C, Alvarez B, Radi R. Inactivation and nitration of human superoxide dismutase (SOD) by fluxes of nitric oxide and superoxide. Free Rad Biol \& Med. 2007; 42:13591368. [PubMed: 17395009]

37. Macmillan-Crow LA, Cruthirds D. Invited review: manganese superoxide dismutase in disease. Free Rad Res. 2011; 34:325-336.

38. Nguyen TT, Stevens MV, Kohr M, Steenberg C, Sack MN, Murphy E. Cysteine 203 of cyclophilin $\mathrm{D}$ is critical for Cyclophilin D activation of the mitochondrial permeability transition pore. J Biol Chem. 2011; 286:40184-40192. [PubMed: 21930693]

39. Han D, Canali R, Rettori D, Kaplowitz N. Effect of glutathione depletion on sites and topology of superoxide and hydrogen peroxide production in mitochondria. Mol Pharmacol. 2003; 64:11361144. [PubMed: 14573763]

40. Batinić-haberle I, et al. Pure MnTBAP selectively scavenges peroxynitrite over superoxide: comparison of pure and commercial MnTBAP samples to MnTE-2-PyP in two models of 
oxidative stress injury, an SOD-specific Escherichia coli model and carrageenan-induced pleurisy. Free Radic Biol Med. 2009; 46:192-201. [PubMed: 19007878]

41. Melov S, et al. A novel neurological phenotype in mice lacking mitochondrial manganese superoxide dismutase. Nat Genetics. 1998; 18:159-163. [PubMed: 9462746] 
A

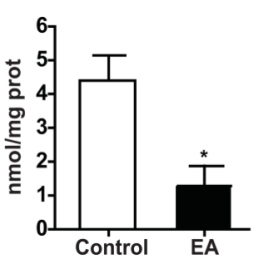

B

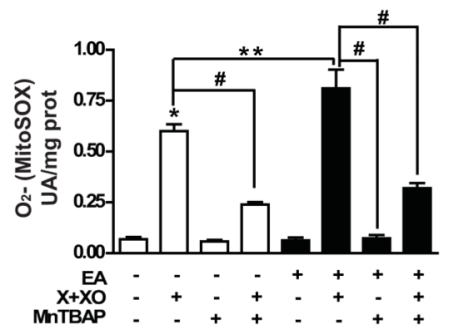

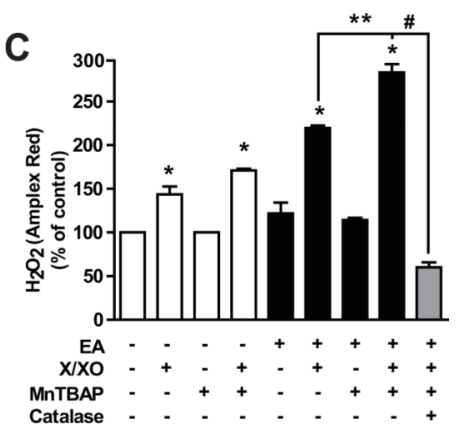

E

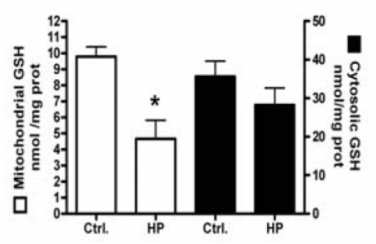

$\mathbf{F}$
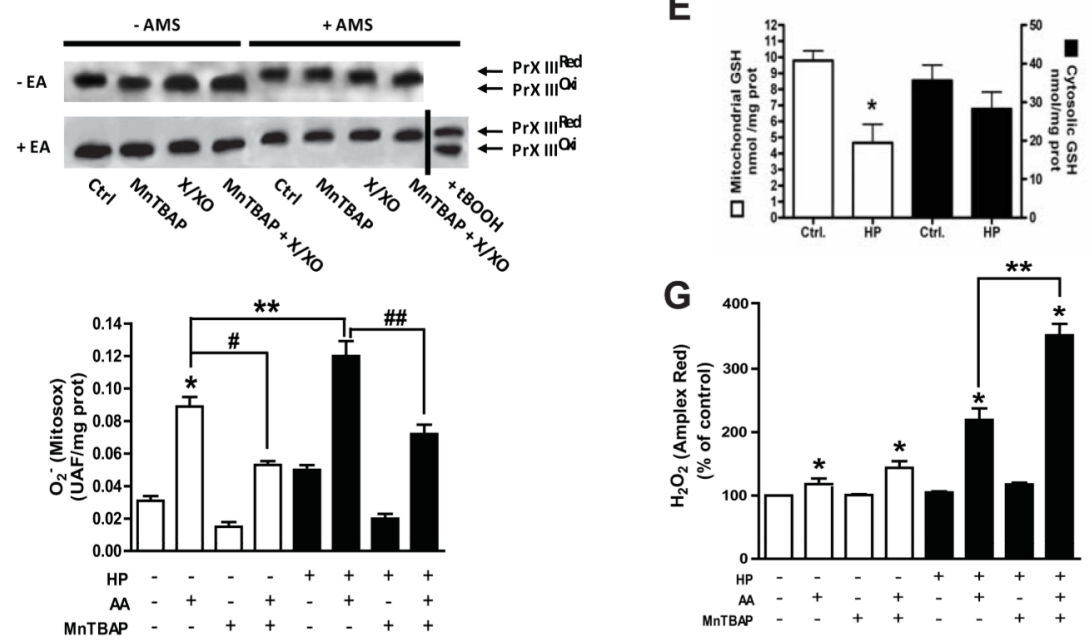

H
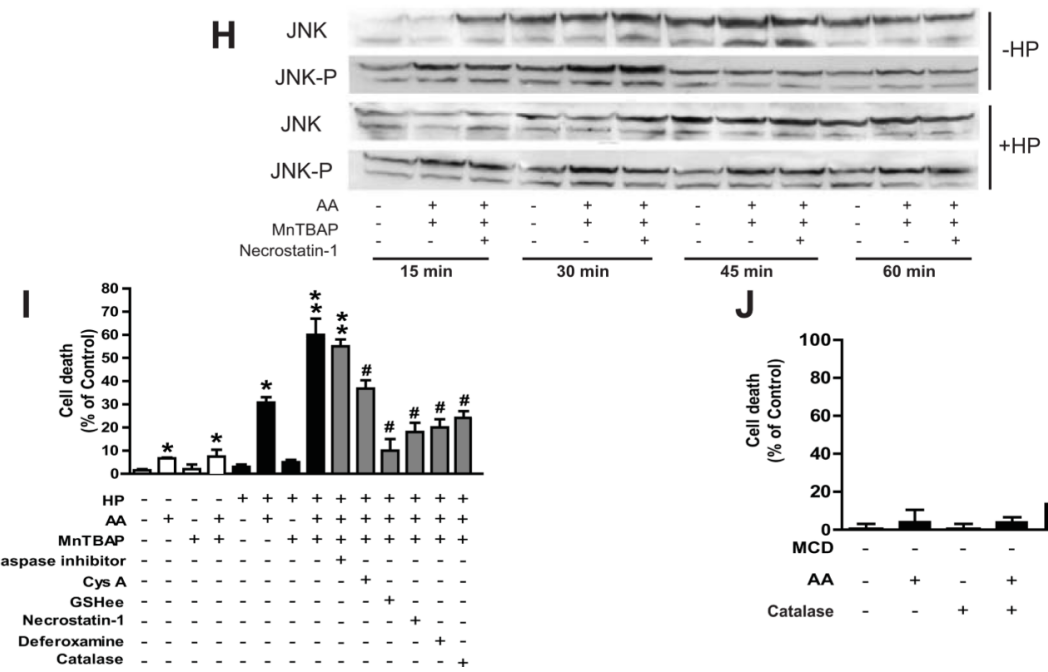

$J$

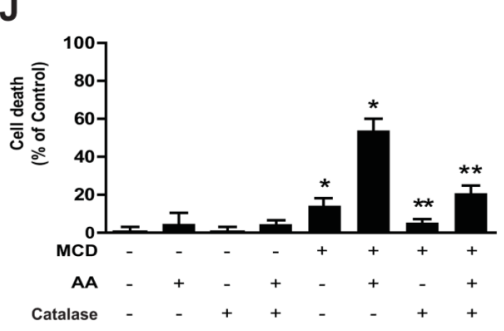

Figure 1. Effect of MnTBAP on isolated rat liver mitochondria with or without GSH depletion and sensitization of mGSH-depleted hepatocytes to SOD mimetic-induced necrotic cell death A, GSH levels after EA incubation; *p<0.05 vs control. B-C, ROS determination by MitoSOX and Amplex Red. Both MitoSOX and Amples Red measurements were done after 10 min of incubation; $\mathrm{B}, * \mathrm{p}<0.05$ vs. control; \# $\mathrm{p}<0.05$ vs $\mathrm{X}+\mathrm{XO} ; * * \mathrm{p}<0.05$ vs $\mathrm{X}+\mathrm{XO}$; \# $\mathrm{p}<0.05$ vs $\mathrm{EA}+\mathrm{X}+\mathrm{XO} ; \mathrm{C},{ }^{*} \mathrm{p}<0.05$ vs control; $* * \mathrm{p}<0.05$ vs $\mathrm{EA}+\mathrm{X} / \mathrm{XO} ; \# \mathrm{p}<0.05$ vs $\mathrm{EA}+\mathrm{X} /$ $\mathrm{XP}+\mathrm{MnTBAP}$. D, redox status of PrX-III in the presence or absence of AMS. tBOOH was used as a positive control to induce the oxidation of Prx-III. Data are the mean \pm SD of 10-12 mitochondrial preparations. E, GSH in cytosol and mitochondria from rat hepatocytes incubated with HP. *p<0.05 vs. control. E-G, ROS determination as in Fig 1B-C following 
incubation with or without AA, HP or MnTBAP; B, *p $<0.05$ vs. control; ** $<<0.05$ vs. AA; \# $\mathrm{p}<0.05$ vs. AA; \#\# $\mathrm{p}<0.05$ vs. HP+AA; C, *p<0.05 vs. control; ** $\mathrm{p}<0.05$ vs. HP+AA. H, Effect of mGSH depletion on the time-dependent JNK phosphorylation after incubation with AA, MnTBAP or necrostatin-1. A representative western blot of 6 individual experiments is shown. I, hepatocellular death determined by Trypan blue and effect of different inhibitors. *p $<0.05$ vs control; \# $\mathrm{p}<0.05$ vs. AA with or without HP; ** $\mathrm{p}<0.05$ vs HP+AA; \#\# $\mathrm{p}<0.05$ vs. HP+AA+MnTBAP. J, cell death of hepatocytes isolated from MCD-fed mice with or without exposure to AA and effect of catalase defense. *p $<0.05$ vs control hepatocytes; $* * p<0.05$ vs. MCD hepatocytes. Data are the mean \pm SD of $12-14$ individual cell preparations. 
A

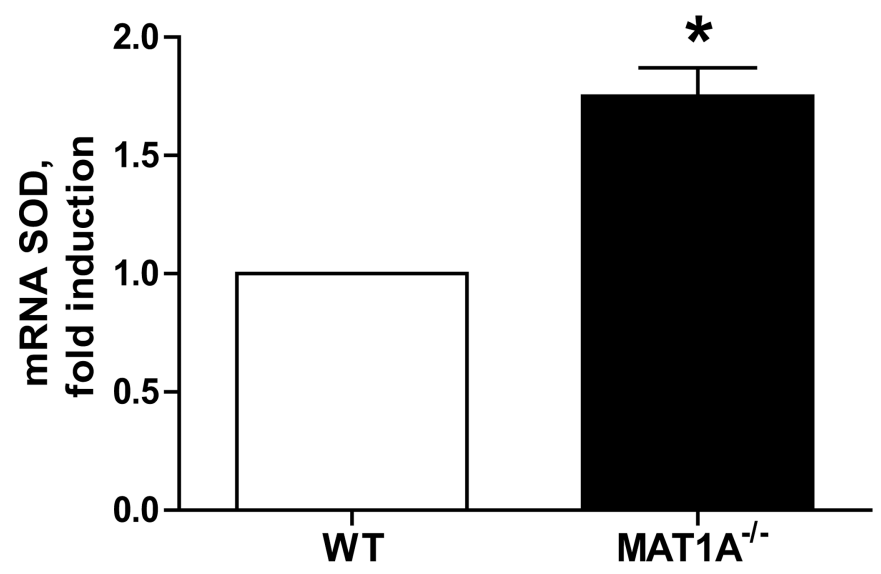

C

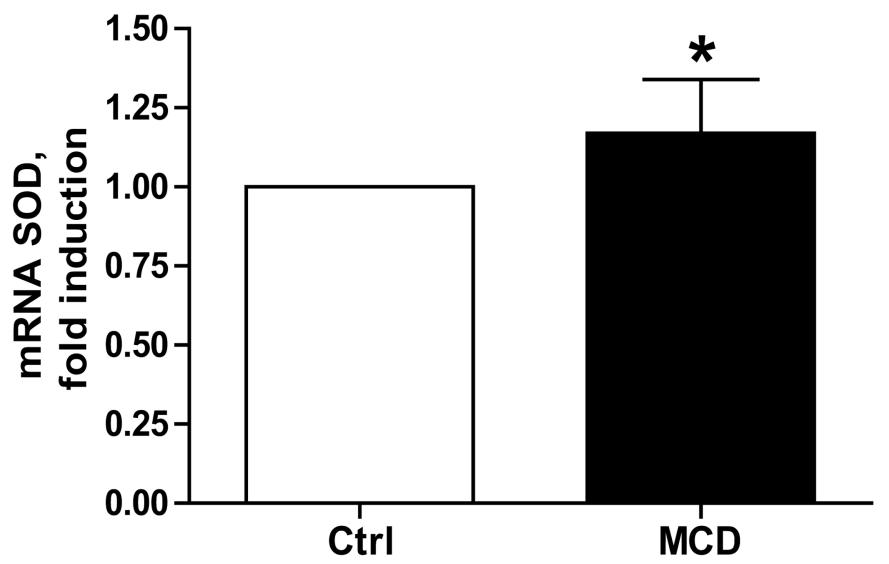

B

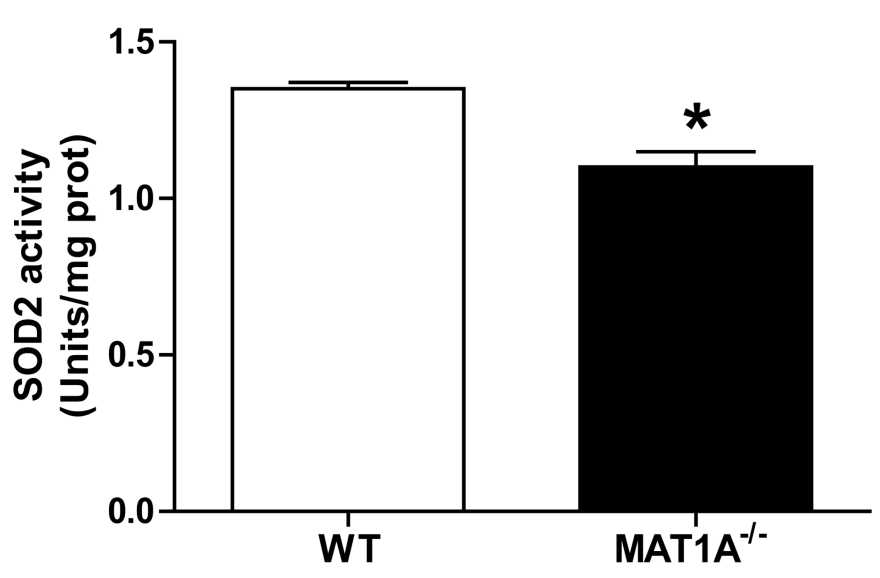

D

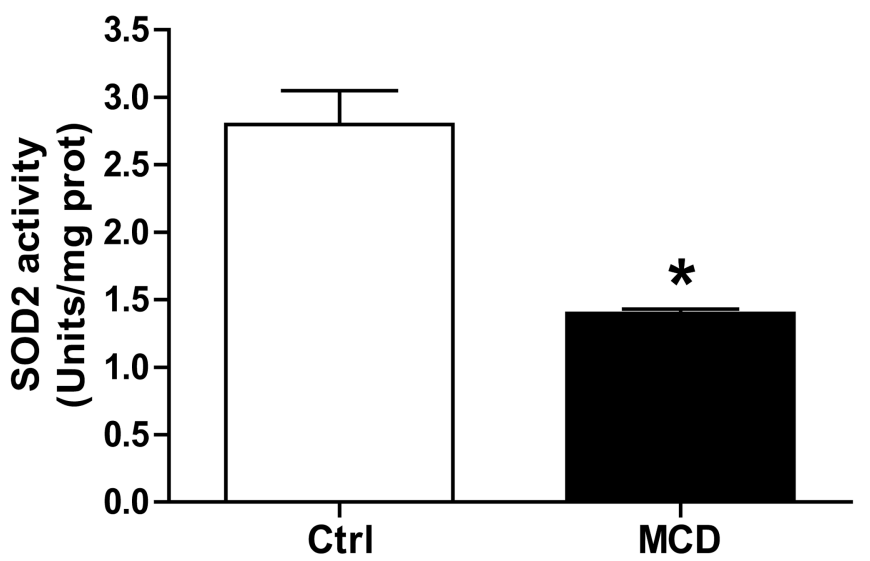

Figure 2. SOD2 expression and activity in experimental models A-B, liver samples from MAT1A KO mice were processed for SOD2 expression (A) and activity (B); ${ }^{*} \mathrm{p}<0.05$ vs control. C-D, liver samples from mice fed MCD diet were processed for SOD2 expression (C) and activity (D); *p<0.05 vs. control. *p<0.05 vs control. Data are the mean \pm SD of 5-6 samples. 
A

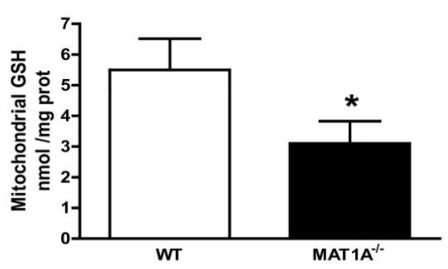

C

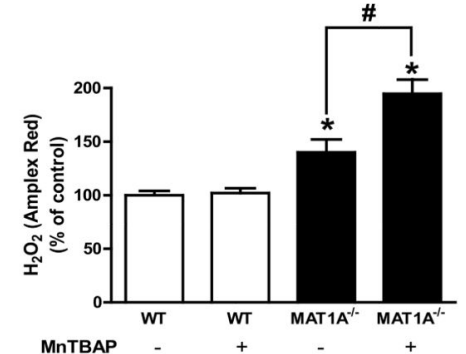

B

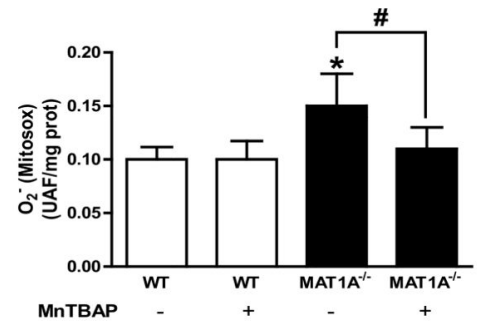

D

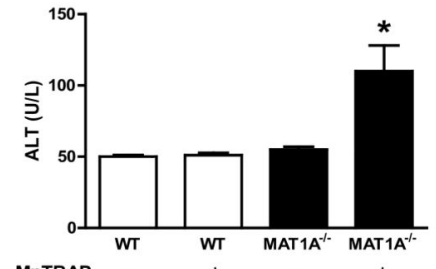

MnTBAP

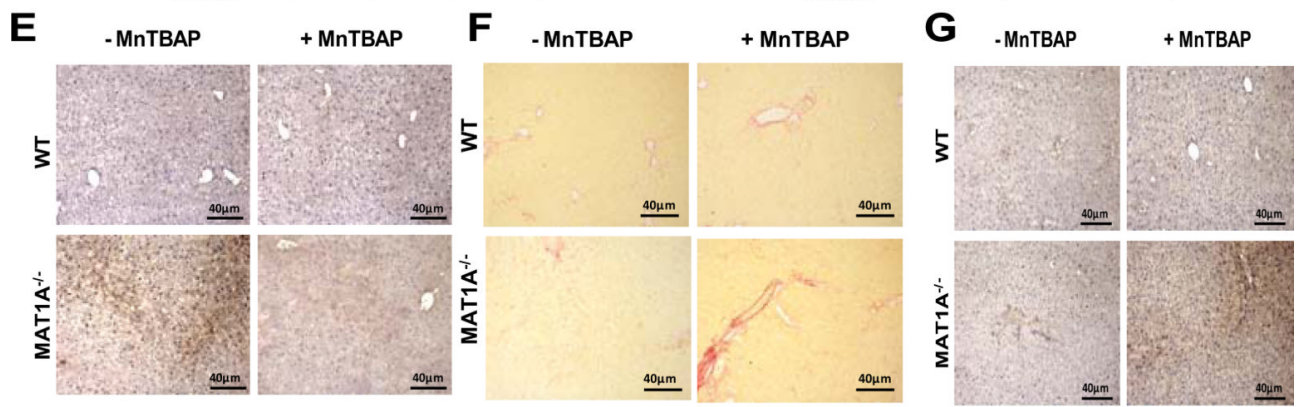

H

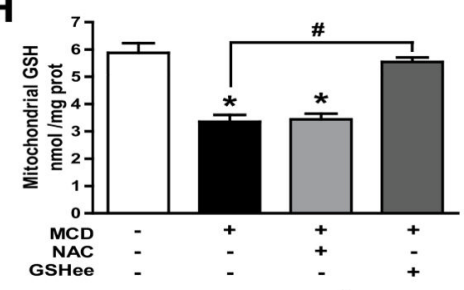

J

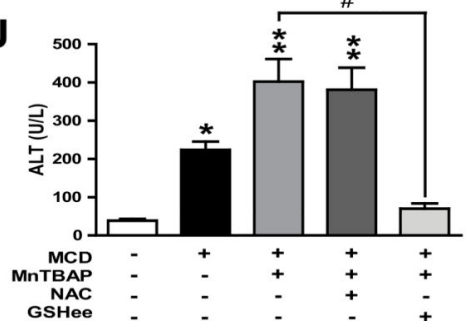

I

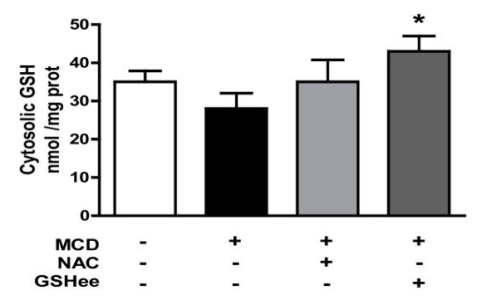

K

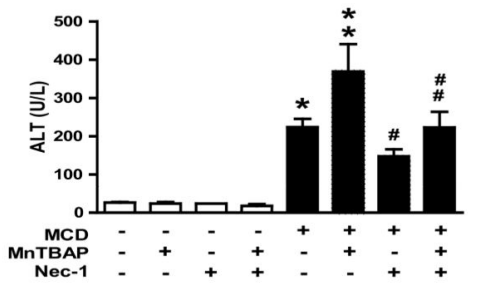

Figure 3. MnTBAP treatment in vivo increases liver injury in MAT1A knockout mice and combination therapy in MCD-fed mice

A, liver mitochondrial GSH from wild type (WT) and MAT1A null mice. B, MitoSOX and C, Amplex Red measurement of liver samples from WT and MAT1A null mice with or without MnTBAP treatment. D, serum ALT levels in WT and MAT1A null mice with or without in vivo treatment with MnTBAP. Representative images of myeloperoxidase, MPO (E), Sirius red (F), and 3-nitrotyrosine immunoreactivity (G) of MAT1A null mice upon MnTBAP treatment. Data are representative images of experiments from 5-6 individual mice. ${ }^{*} \mathrm{p}<0.05$ vs. WT and MAT1A null mice; \#p $<0.05$ vs. MAT1 $\mathrm{A}^{-/-}$mice. H-I, hepatic mitochondrial GSH (H) or cytosol GSH (I) from MCD-fed mice with or without in vivo 
treatment with NAC or GSHee; *p $<0.05$ vs control; \#p $<0.05$ vs. MCD. J-K, serum ALT levels from MCD fed mice following treatment with MnTBAP, NAC, GSHee or necrostatin-1 (Nec-1) as indicated; *p<0.05 vs. control; **p<0.05 vs MCD; \#p<0.05 vs. MCD+MnTBAP;\#\#p<0.05 vs. MCD+MnTBAP. Data are the mean \pm SD of 4-6 individual mice. 
A

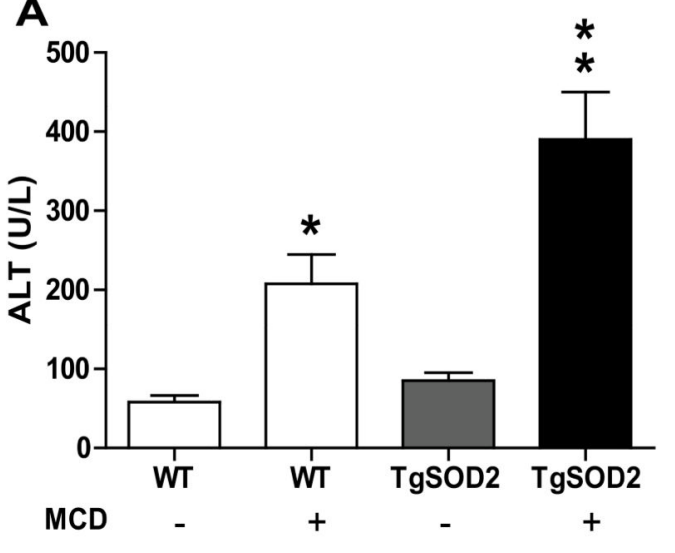

B

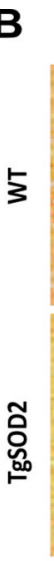

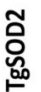

- MCD
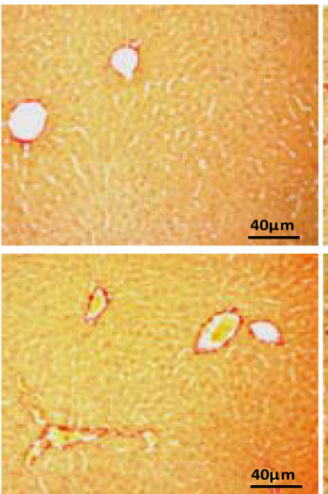

+ MCD

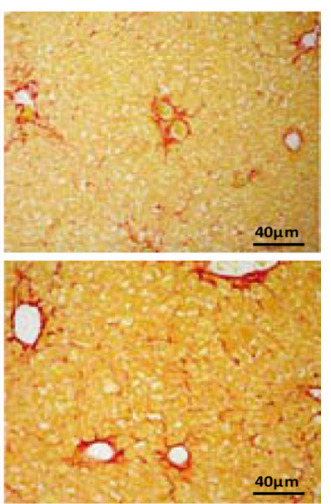

C

- MCD

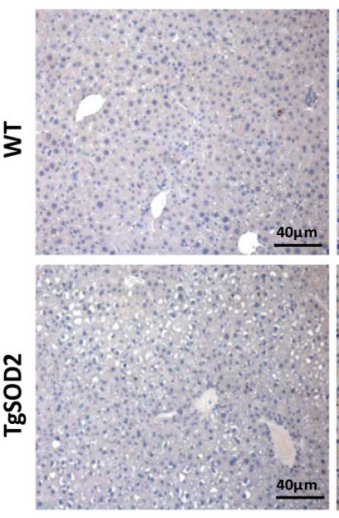

+ MCD

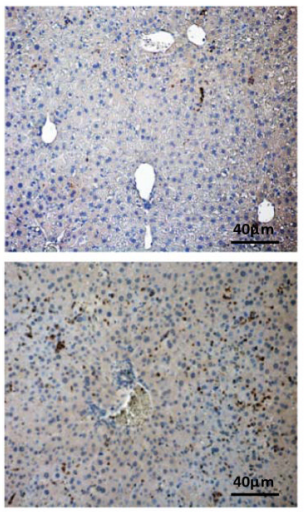

D
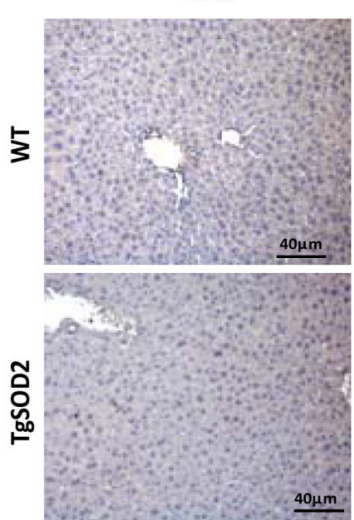

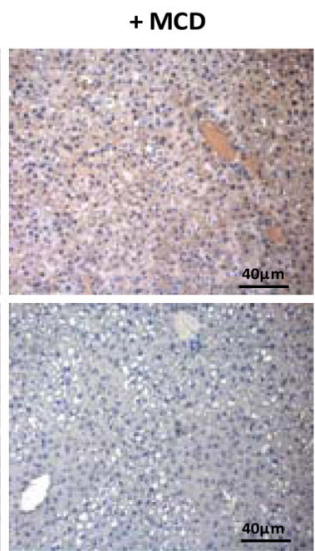

E
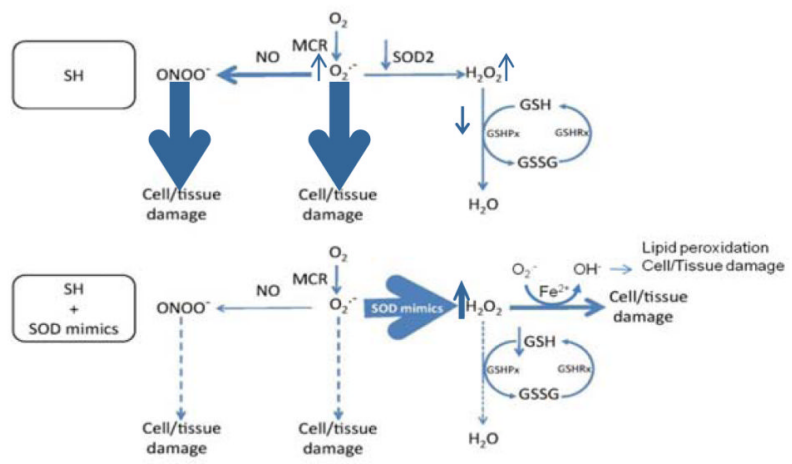

Figure 4. TgSOD2 mice exhibit increased susceptibility to MCD-induced SH

A, serum ALT levels from wild type mice or TgSOD2 mice fed MCD; *p<0.05 vs. WT; $* * p<0.05$ vs. WT+MCD. B, Sirius red staining of liver sections from wild type mice or TgSOD2 mice fed MCD diet. Data are the mean \pm SD of 3-4 mice. C, myeloperoxidase staining of liver samples from wild type mice or TgSOD2 mice fed MCD diet. D, 3nitrotyrosine immunoreactivity of wild type mice and TgSOD2 mice fed MCD diet. E, schematic diagram illustrating the effects of superoxide scavenging by SOD mimics with or without GSH depletion. 